\title{
AN ANALYSIS OF SPONTANEOUS RECOMBINATION IN DROSOPHILA MELANOGASTER MALES
}

\author{
ISOLATION AND CHARACTERISATION OF MALE \\ RECOMBINATION LINES
}

\author{
R. C. WOODRUFF* \\ Department of Genetics, University of Cambridge, Cambridge CB4 1XH, England \\ and \\ J. N. THOMPSON, Jr. \\ Department of Zoology, University of Oklahoma, Norman, Oklahoma 73019, U.S.A.
}

Received 20.vii.76

\section{SUMMARY}

Spontaneous second chromosome recombination has been observed in males of Drosophila melanogaster newly derived from natural populations. From a Cambridge, England, natural population, two lines out of 20 showed male recombination, and in an Oklahoma, U.S.A., natural population all of 25 lines showed male recombination. One line, $O K 1$, was characterised in more detail, and it was observed that male recombination was probably caused by dominant second and dominant third chromosome elements. The majority of recombination events in the second chromosome occurred in the centromeric region and in the right arm, and male recombinants were recovered in clusters, suggesting that some male recombination occurs in premeiotic cells.

Since both single and multiple recombinants were recovered, it was possible to measure chromosome interference for male recombination. This interference was observed to be negative. Finally, there is a reciprocal-cross effect associated with male recombination and $F 1$ female sterility in the $O K 1$ line. This effect on male recombination is apparently not caused by a cytoplasmic suppressor of recombination.

The genetic basis and mechanism of male recombination are discussed, along with its possible influence on adaptability in natural populations.

\section{InTRODUCTION}

Morgan (1912, 1914) was the first to report that spontaneous recombination does not occur in male Drosophila melanogaster. The absence of spontaneous male recombination formed the basis of elegant breeding programmes and was an accepted rule-of-thumb for almost 50 years until Hiraizumi (1971) reported that a low, but significant, frequency of recombination occurred in males captured from a natural population in southern Texas. Although it was originally thought that this population was exceptional, it is now becoming clear that strains of $D$. melanogaster that show male recombination are not rare. Such strains have been identified in 10 states of the U.S.A. (Hiraizumi, 1971; Voelker, 1974; Waddle and Oster, 1974; Yamaguchi and Mukai, 1974; Kidwell and Kidwell, 1975a, 1975b; Slatko, personal communication), in Australia (Sved, 1974, 1976), in Greece (Pelecanos and Yannopoulos, personal communication), and in Yugoslavia, Japan, and

* Present address : Department of Zoology, University of Oklahoma, Norman, Oklahoma 73019, U.S.A. 


\title{
AN ANALYSIS OF SPONTANEOUS RECOMBINATION IN DROSOPHILA MELANOGASTER MALES
}

\author{
ISOLATION AND CHARACTERISATION OF MALE \\ RECOMBINATION LINES
}

\author{
R. C. WOODRUFF* \\ Department of Genetics, University of Cambridge, Cambridge CB4 1XH, England \\ and \\ J. N. THOMPSON, Jr. \\ Department of Zoology, University of Oklahoma, Norman, Oklahoma 73019, U.S.A.
}

Received 20.vii.76

\section{SUMMARY}

Spontaneous second chromosome recombination has been observed in males of Drosophila melanogaster newly derived from natural populations. From a Cambridge, England, natural population, two lines out of 20 showed male recombination, and in an Oklahoma, U.S.A., natural population all of 25 lines showed male recombination. One line, $O K 1$, was characterised in more detail, and it was observed that male recombination was probably caused by dominant second and dominant third chromosome elements. The majority of recombination events in the second chromosome occurred in the centromeric region and in the right arm, and male recombinants were recovered in clusters, suggesting that some male recombination occurs in premeiotic cells.

Since both single and multiple recombinants were recovered, it was possible to measure chromosome interference for male recombination. This interference was observed to be negative. Finally, there is a reciprocal-cross effect associated with male recombination and $F 1$ female sterility in the $O K 1$ line. This effect on male recombination is apparently not caused by a cytoplasmic suppressor of recombination.

The genetic basis and mechanism of male recombination are discussed, along with its possible influence on adaptability in natural populations.

\section{InTRODUCTION}

Morgan (1912, 1914) was the first to report that spontaneous recombination does not occur in male Drosophila melanogaster. The absence of spontaneous male recombination formed the basis of elegant breeding programmes and was an accepted rule-of-thumb for almost 50 years until Hiraizumi (1971) reported that a low, but significant, frequency of recombination occurred in males captured from a natural population in southern Texas. Although it was originally thought that this population was exceptional, it is now becoming clear that strains of $D$. melanogaster that show male recombination are not rare. Such strains have been identified in 10 states of the U.S.A. (Hiraizumi, 1971; Voelker, 1974; Waddle and Oster, 1974; Yamaguchi and Mukai, 1974; Kidwell and Kidwell, 1975a, 1975b; Slatko, personal communication), in Australia (Sved, 1974, 1976), in Greece (Pelecanos and Yannopoulos, personal communication), and in Yugoslavia, Japan, and

* Present address : Department of Zoology, University of Oklahoma, Norman, Oklahoma 73019, U.S.A. 
Taiwan (Yamaguchi, 1976). Our report extends this list to include Oklahoma, U.S.A., and Cambridge, England. Furthermore, the presence of male recombination is not peculiar to $D$. melanogaster; spontaneous male recombination is known for $D$. ananassae (for a review, see Moriwaki and Tobari, 1975), D. simulans (Woodruff and Bortolozzi, 1976), D. subobscura (Phillips, 1944), D. virilis (Kikkawa, 1935), and D. willistoni (Franca, Da Cunha and Garrido, 1968).

If male recombination does occur regularly in natural populations, and is not just an exceptional event, it may provide an additional factor affecting variability in such populations, and warrants detailed analysis. In particular, the mechanism and genetic basis of male recombination need to be identified. In this respect, the following key points have been reported for D. melanogaster: male recombination is under the influence of dominant (or semidominant) elements located on the second and/or third chromosomes (Voelker, 1974; Slatko and Hiraizumi, 1975). The distribution of male recombination events is different from that of female recombination events (Hiraizumi et al., 1973; Voelker, 1974; Kidwell and Kidwell, 1975b), and at least some of the male recombination events are premeiotic in origin (Hiraizumi et al., 1973; Voelker, 1974; Kidwell and Kidwell, 1975b). Mutator activity, segregation distortion, and sterility seem to be associated with male recombination in some lines (Slatko and Hiraizumi, 1973; Sved, 1974, 1976; Voelker, 1974; Kidwell and Kidwell, 1975b; Yamaguchi, 1976). Finally, there seems to be a cytoplasmic-chromosomal interaction that can affect the frequency of male recombination (Kidwell and Kidwell, 1975a).

We have now determined the frequencies of male recombination associated with chromosomes that were isolated from two widely separated populations, one from the U.S.A. and one from England, and have found male recombination in both. In this report we define male recombination simply as an exchange of markers without implying a mechanism for the exchange. The genetic characterisation of one of these lines, $O K 1$, is reported here.

A preliminary note on some of this data has been published elsewhere (Woodruff and Thompson, 1975).

\section{Materials and methods}

\section{(i) Stocks}

The mutant genes, chromosomal rearrangements, wild-type stocks, and special chromosomes used in this study are listed in table I. All stocks were maintained at $25 \pm 2{ }^{\circ} \mathrm{C}$ on a standard oatmeal, molasses, agar, nipagin medium.

\section{(ii) Natural populations}

Two natural populations of $D$. melanogaster were screened for male recombination. One was from Oklahoma City, Oklahoma, U.S.A. (assigned the symbol $O K$ ) and one from Cambridge, England (assigned the symbol $C W$ ). Both male and female adults were captured in watermelon traps in Oklahoma from one location in I day (20th October 1970). These flies were airmailed to Cambridge, England, where the progeny of individual females were kept by mass transfers as separate lines. Adult males were captured from the $C W$ population from one location over a 9-day period 
TABLE 1

Mutants, chromosome aberrations, wild-type stocks, and special chromosomes (Lindsley and Grell, 1968)

\begin{tabular}{ll}
$\quad$ Symbol & \multicolumn{1}{c}{ Name (structure affected) } \\
$b$ & black (body colour) \\
$b w$ & brown (eye colour) \\
$c n$ & cinnabar (eye colour) \\
$c n^{2}$ & cinnabar-2 (eye colour) \\
$C y$ & Curly (wing shape) \\
$d p$ & dumpy (wing shape) \\
$d p^{1 V I}$ & dumpy-lethal vortex of Ives \\
& $\quad$ (wing shape) \\
$e^{8}$ & ebony-sooty (body colour) \\
$G l$ & Glued (eye texture) \\
$G l a$ & Glazed (eye texture) \\
& \\
$H$ & Hairless (bristles) \\
$p r$ & purple (eye colour) \\
$S b$ & Stubble (bristle length) \\
Ser & Serrate (wing shape) \\
$v e$ & veinlet (wing veins) \\
In $(2 L+2 R)$ Cy & Inversion (2L+2R) Curly \\
In $(2 L R)$ CyO & Inversion (2LR) Curly of Oster \\
In $(3 L R)$ Ubx $x^{130}$ & Inversion (3LR) Ultrabithorax-130 \\
& (halter size) \\
Canton-S & Canton-Special \\
$C W$ & Cambridge-Wild \\
OK & Oklahoma
\end{tabular}

$2 \mathrm{~L}-48 \cdot 0$

$2 \mathrm{R}-104 \cdot 5$

$2 \mathrm{R}-57 \cdot 5$

$2 \mathrm{R}-57 \cdot 5$

$2 \mathrm{~L}-6 \cdot 1$, recessive lethal

$2 \mathrm{~L}-13 \cdot 0$

$2 \mathrm{~L}-13 \cdot 0$

$3 \mathrm{R}-70 \cdot 7$

$3 \mathrm{~L}-4 \mathrm{l} \cdot 4$, recessive lethal

2nd chromosome inversions, recessive lethal, balancer

$3 R-69 \cdot 5$, recessive lethal

$2 \mathrm{R}-54 \cdot 5$

$3 \mathrm{R}-58 \cdot 2$, recessive lethal

$3 \mathrm{R}-92 \cdot 5$, recessive lethal

$3 \mathrm{~L}-0 \cdot 2$

2nd chromosome inversions, balancer

2nd chromosome inversions, balancer

3rd chromosome inversions, balancer

wild-type stock

wild-type stock

wild-type stock

(11th-19th September 1974). Bananas and apples were used as bait, and males were captured with a suction tube. $C W$ males were mated individually to $d p b c n b w ; v e$ females and lines were maintained by backcrossing heterozygous males to homozygous $d p b \mathrm{cn} b w ; v e$ females. All of the $O K$ and $C W$ lines were initially screened for male recombination in October 1974.

\section{Results}

(i) Initial screen for male recombination

The following crosses were performed as an initial screen for spontaneous male recombination.

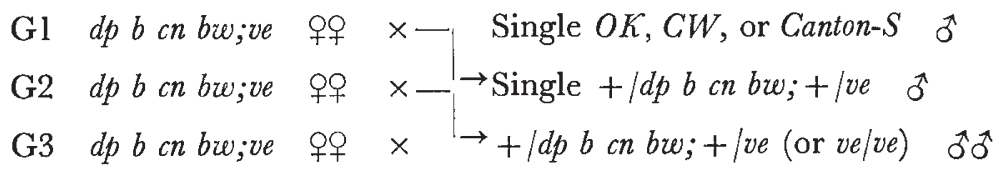

All G4 progeny were scored for the occurrence of second-chromosome male recombination. Due to the difficulty of differentiating newly emerged $b$ and $b^{+}$flies, no double recombinants of the genotypes $d p^{+} b \mathrm{cn}^{+} b w^{+}$and $d p b^{+} c n b w$ were scored; the body colour phenotype was determined in all other progeny. In this screening programme the effect of the small fourth chromosome was ignored, since by repeated backcrosses to the $d p b$ cn bw;ve stock without selection for the fourth chromosome, the fourth chromosomes in G3 and subsequent crosses were probably from the $d p b c n$ bw;ve stock. 


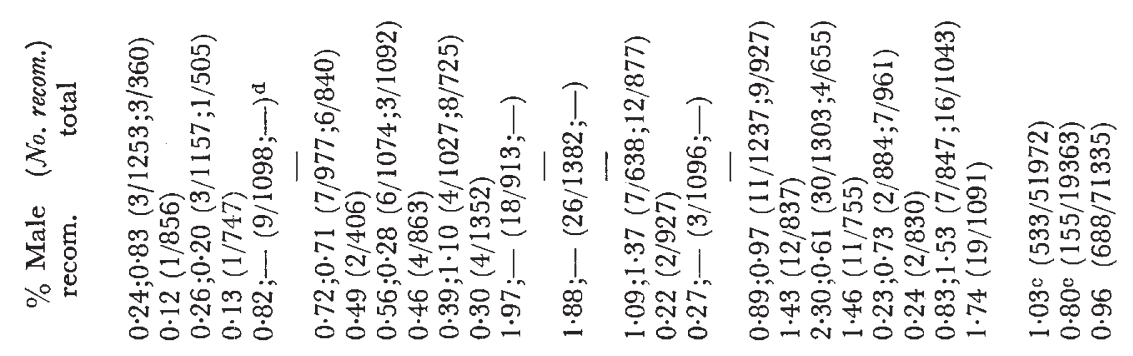
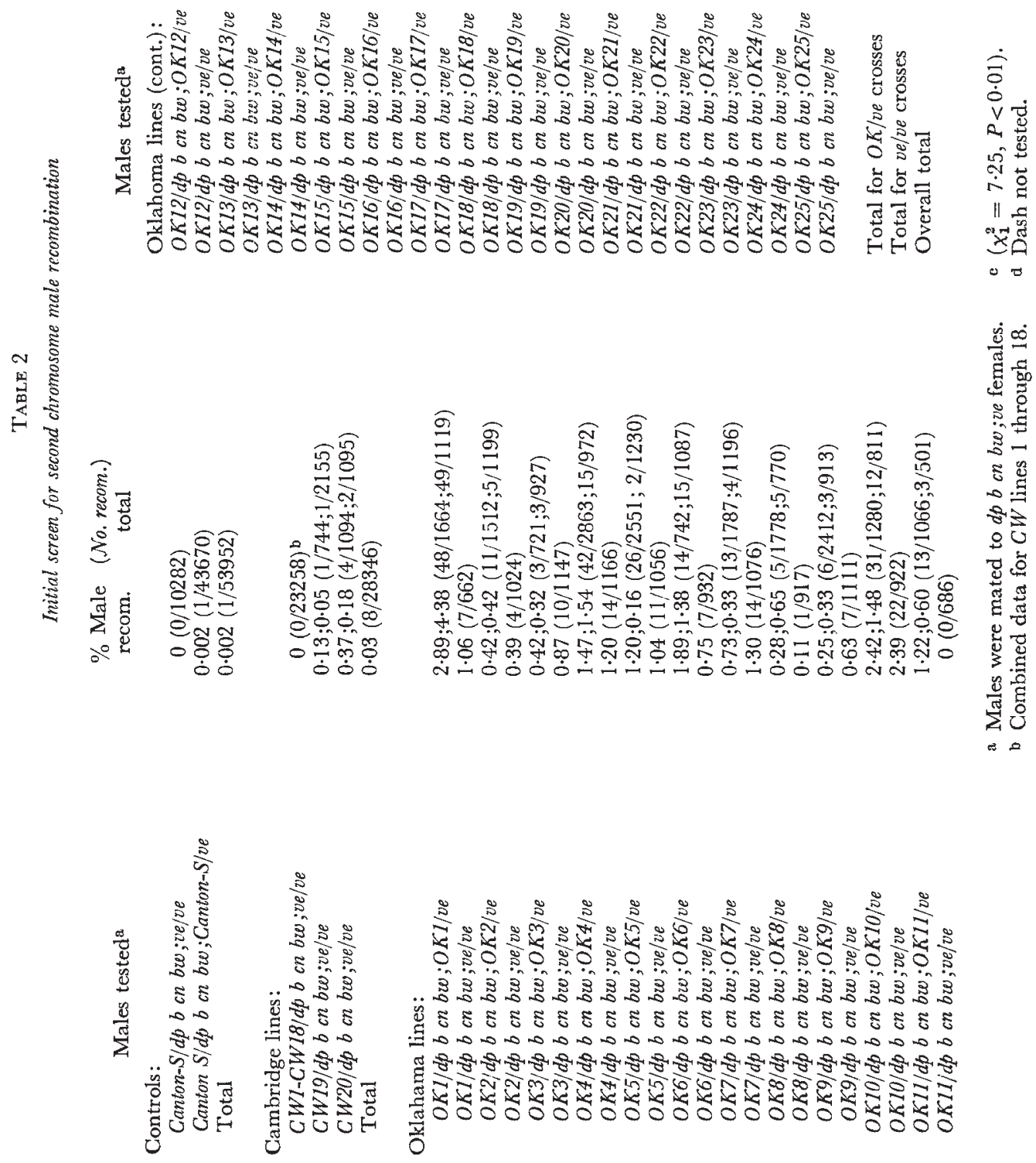
Each bottle of the G3 cross was set up with five males and ten females and, after 4 to 5 days, the parents were transferred once. The G4 progeny were scored no later than 18 days after the G3 crosses were initiated. This eliminated the possibility of scoring G5 female recombination progeny as G4 male recombination progeny.

\section{(ii) Control, $C W$ and $O K$ crosses}

Two control crosses were performed using the Canton- $S$ wild-type stock. From G3 crosses using Canton-S/dp b cn bw;ve/ve males, no male recombinants were observed among 10,282 progeny, and in G3 crosses using Canton-S/dp $b$ cn bw; Canton-S/ve males, only 1 male recombinant $\left(d p^{+} b^{+} c n b w\right)$ was observed out of 43,670 progeny (overall total $=1 / 53,952 ; 0.002$ per cent).

The absence (or very low frequency) of male recombination has been reported for Canton-S (Kidwell and Kidwell, 1975a), as well as for other laboratory wild stocks-including Oregon-R-C, Princeton, Ames I (Kidwell and Kidwell, 1975a) and Tokoyo (Slatko and Hiraizumi, 1973). The absence of male recombination in these Canton-S/dp $b$ cn bw heterozygotes suggests that the $d p b \mathrm{cn} b w ; v e$ chromosomes do not themselves induce male recombination.

The second chromosomes of the Canton-S stock used in these control crosses were free of any gross chromosomal rearrangements. This was determined by salivary gland chromosome analysis and by the recovery of normal frequencies of recombination in Canton-S/dp $b$ cn bw females (see table 3).

$C W$ and $O K$ crosses. Table 2 shows the male recombination frequencies observed for $20 \mathrm{CW}$ lines and for $25 \mathrm{OK}$ lines. Two of the $C W$ lines (CW19 and $C W 20$ ) and all of the $O K$ lines showed male recombination. Further tests using 23 of the lines showing male recombination indicated that the factors involved were inherited since all of the lines again had male recombination. The frequencies of male recombination in these two populations are similar to those reported by Hiraizumi (1971), Kidwell and Kidwell

TABLE 3

Recombination maps of the second chromosome

$\begin{array}{llll}\begin{array}{c}O K 1 / d p b \text { cn bw male- } \\ \text { derived map }\end{array} & & & \\ \begin{array}{c}\text { Uncorrected for large } \\ \text { clusters (see table } 4)\end{array} & 0.13(33 / 25597) & 0.55(141 / 25597) & 1.31(336 / 25597) \\ \begin{array}{c}\text { Corrected for large } \\ \text { clusters }\end{array} & 0.13(33 / 25418) & 0.55(141 / 25418) & 0.62(157 / 25418) \\ \begin{array}{c}\text { Canton-S/dp } b \text { cn bw } \\ \text { male-derived map }\end{array} & 0(0 / 53952) & 0.002(1 / 53952) & 0(0 / 53952) \\ \begin{array}{c}\text { OK1/dp } b \text { cn bw } \\ \text { female-derived map }\end{array} & 30 \cdot 2(559 / 1849) & 11.6(215 / 1849) & 34.2(633 / 1849) \\ \begin{array}{c}\text { Canton-S/dp } b \text { cn bw } \\ \text { female-derived map }\end{array} & 26.4(316 / 1198) & 3.0(36 / 1198) & 30 \cdot 1(360 / 1198) \\ \begin{array}{c}\text { Standard female-derived } \\ \text { map (Lindsley and Grell, } \\ \text { 1968) }\end{array} & 35.5 & & 47.0 \\ & \text { a Pooled data from tables } 2 \text { and } 4 . & \end{array}$


(1975a), Voelker (1974), and Waddle and Oster (1974), and are significantly higher than our controls.

To insure that these male recombinants were true recombinants, a sample of 282 presumptive recombinant males were mated to $d p b \mathrm{cn} b w ; v e$ virgin females. All males were found to breed as true second-chromosome recombinants. In addition, we can conclude that the male recombination observed in these crosses is probably not due to a specific interaction between the wild population genome and the $d p b$ cn bw;ve genome. This was shown by recovering male recombinants from a cross with a marker stock other than $d p b$ cn bw; ve, i.e. from crosses of $O K 1 / c n$ bw males to $c n$ bw females, 0.3 per cent (4/1333) male recombination was observed between $c n$ and $b w$.

Unlike some of the previous reports of male recombination, we observed a number of double recombination events: $19 d p^{+} b^{+} c n b w^{+}$and two $d p b c n^{+} b w$ double recombinants were recovered. In one line $(O K 10)$ eight $d p^{+} b^{+} c n b w^{+}$double recombinants were observed from one bottle, i.e. from the progeny of five males. This observation suggests that some of the male recombination events may occur in clusters and may be premeiotic in origin.

Hiraizumi (197I) has reported that segregation distortion is associated with male recombination in some lines. The amount of segregation distortion was measured by the value $k$, where $k$ is the proportion of nonrecombinant $+/$ marker progeny from crosses of $+/$ marker males to homozygous marker females. A value of $k=0.5$ indicates no distortion. There seems to be no clear indication of segregation distortion for the second chromosomes in the $C W$ lines that show male recombination (CW19 and $C W 20)$ or in the $O K$ lines. The total $k$ values for male recombination in the initial screens were: $0.55(2809 / 5080)$ for the CW19 and CW20 lines, $0.56(39243 / 70685)$ for the $O K$ lines, and 0.57 (30897/5395I) for the Canton-S line. No $k$ values were large enough to suggest that chromosomes carried Segregation Distorter $(S D)$.

\section{(iii) Chromosome location of the male recombination elements}

It can be concluded from the data in table 2 that the element(s) which causes recombination in the $C W / d p b c n$ bw;ve/ve males is dominant or semidominant and is located on the second chromosome. This conclusion is based on the observation that the ve third chromosome does not induce male recombination (table 2).

On the other hand, because the $O K$ lines were originally tested as heterozygotes for the second and the third chromosomes, the dominant malerecombination element(s) in these lines could be located on the second, third, or on both chromosomes. The chromosomal location of this element was, therefore, determined by crossing $2 \mathrm{I}$ different $O K / d p b c n$ bw; ve/ve males (each containing only a single wild second chromosome) to $d p b$ cn bw;ve females and scoring for male recombination. If the element is located only on the $O K$ third chromosomes, then male recombination should be absent from these crosses. Table 2 shows the results of these crosses. In all but one line male recombination was present, although the total frequency of recombination was significantly lower than the total frequency from $O K / d p b c n b w$; $O K / v e$ males $\left(\chi_{1}^{2}=7 \cdot 3, P<0.01\right)$. An analysis of the distribution of male recombination along the second chromosome in the presence and absence of the $O K$ third chromosomes shows that there is a significant difference 
$\left(\chi_{1}^{2}=16 \cdot 2, P<0.001\right)$ in the frequencies of male recombination in only the $b$-cn region (in $O K / d p b c n b w ; O K / v e$ males, $d p-b=36 / 51972=0.07$ per cent, $b$-cn $=250 / 51972=0.48$ per cent, $c n-b w=247 / 51972=0.48$ per cent; in $O K / d p b$ cn bw; ve/ve males, $d p-b=17 / 19363=0.09$ per cent, $b$ - $c n=50 / 19363=0.26$ per cent, $c n-b w=88 / 19363=0.45$ per cent $)$. The overall reduction in the frequency of male recombination is probably due to the removal of a third-chromosome element(s) in the $O K / d p b \mathrm{cn}$ bw; ve/ve male that has a minor influence on male recombination.

As in the $O K / d p \quad b \quad n \quad b w ; O K / v e$ crosses, double recombinants were observed from these crosses. A total of $12 d p^{+} b^{+} c n b w^{+}$and two $d p b c n^{+} b w$ double recombinants were recovered, with one line $(O K 10$, the same line that had a double recombinant presumptive cluster in the $O K / d p b \mathrm{cn} b w$; $O K / v e$ crosses) giving seven $d p^{+} b^{+} c n b w^{+}$events from one bottle.

The overall results from $O K / d p b c n b w ; O K / v e$ and $O K / d p b c n$ bw; velve crosses show that there is an equal recovery of reciprocal exchange events in the $b-c n$ and $c n$ - $b w$ regions but not in the $d p-b$ region. The total numbers of single recombinants in the $c n$-bw region $\left(d p b c n b w+=136\right.$ and $d p^{+} b^{+} c n^{+}$ $b w=166)$ and in the $c n$-bw region $\left(d p b c n^{+} b w^{+}=125\right.$ and $d p^{+} b^{+} c n b w=$ 139) are not significantly different from a ratio of $1: 1\left(b-c n, \chi_{1}^{2}=3 \cdot 0\right.$, $\left.P>0.05 ; b-c n, \chi_{1}^{2}=0.7, P>0.05\right)$. However, in the $d p-b$ region the numbers of male recombinants $\left(d p b^{+} c n^{+} b w^{+}=41\right.$ and $\left.d p^{+} b c n b w=12\right)$ are significantly different from a $1: 1$ ratio $\left(\chi_{1}^{2}=15.9, P<0.001\right)$. We have not tested the possibility that there are viability differences in the latter two genotypes.

Since all of the $C W$ and $O K$ lines with male recombination gave similar results, we decided to concentrate the rest of this study on one line-that having the highest male recombination frequency, $O K 1$.

Using the OK1 line, we crossed Canton-S/dp b cn bw;OK1/ve males with $d p b$ cn bw;ve females and scored progeny for recombination in males. Since it has been shown by the control crosses that the Canton- $S$ second chromosome does not induce male recombination, any male recombination from this cross would have to be due to an influence of the $O K 1$ third chromosome. From this cross, eight recombinants were observed out of 4303 progeny $(0.19$ per cent $)$. This frequency is significantly lower than the frequency from $O K 1 / d p$ b cn bw;OK1/ve and $O K 1 / d p$ b cn bw;ve/ve males $(108 / 4469=$ 2.42 , table 2) $\left(\chi_{1}^{2}=81.9, P<0.001\right)$ and is significantly higher than the controls $(P<0.001)$. The major effect on male recombination in the $O K 1$ line is, therefore, due to a dominant or semi-dominant second-chromosome element, which is influenced by a dominant or semi-dominant thirdchromosome element. The OK1 Y chromosome does not influence the frequency of male recombination events (Woodruff, unpublished).

We are not yet able to give a map position for this second-chromosome male recombination element, although we do have preliminary data which suggests that it is probably located on $2 \mathrm{R}$ between $c n(57.5)$ and bw (104.5).

\section{(iv) Characterisation of male recombination in the OKI line}

(a) Male- and female-derived recombination maps. Table 3 presents recombination maps derived from male and female second-chromosome recombination in $O K 1 / d p b c n$ bw and in Canton-S/dp b cn bw (control) lines. In the presence of $O K 1$, about 94 per cent of all second-chromosome male 
recombination occurs in the area from $b$ to $b w$, i.e. in the region near the centrometric heterochromatin and in the right arm. This is especially evident when one compares the $O K 1 / d p b$ cn bw male-derived map to the $0 K 1 / d p b$ cn bw female-derived map (fig. 1). In particular, about 42 per cent of the male recombination events occur between $b$ and $c n$, whereas only 3 per cent of the female recombination events occur in this region. Similar distributions of male recombination events have been observed in D. melanogaster (Hiraizumi, et al., 1973; Voelker, 1974; Kidwell and Kidwell, 1975b). There has also been observed a difference in the distribution of female and male recombination in the Phorid, Megaselia scalaris (Mainx, 1964).

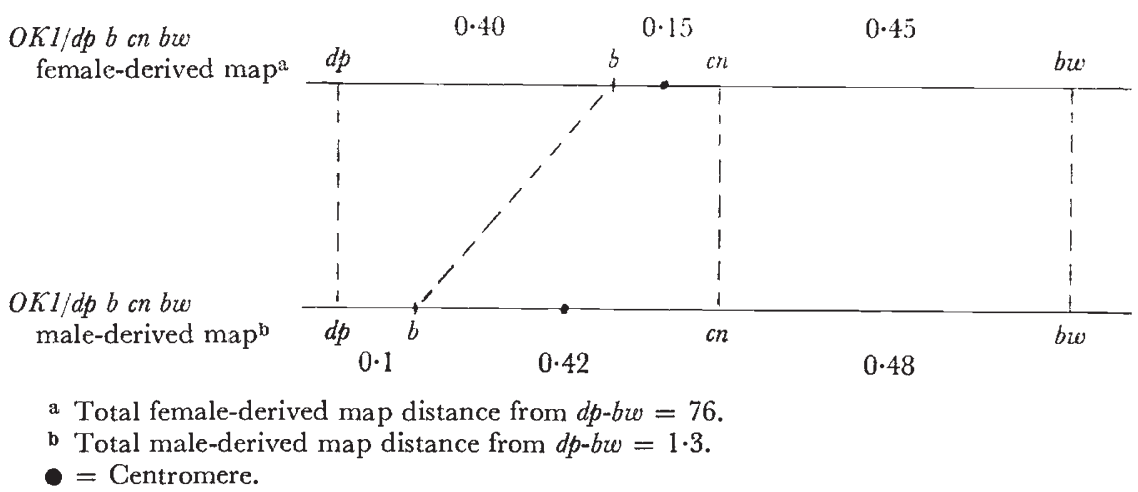

Fig. 1.-A comparison of the male- and female-derived second chromosome maps from $O K 1 / d p b$ cn bw flies (expressed as fractions of the total recombination distances) (corrected for large cluster events)

(b) Time of male-recombination events. A determination of the time (germ cell stage) at which male recombination occurs can give an insight into the mechanism of male recombination. In particular, one can determine whether events occur in mitotic germ cells or in meiotic cells. In this respect, it has been shown (Kale, 1969; Hiraizumi et al., 1973) that if male recombination events occur only in meiotic cells (spermatocytes) no clusters of recombinants occur and these events fit a Poisson distribution. Conversely, premeiotic events give clusters of male recombinants that do not fit a Poisson distribution. Using this rationale, the best previously published data suggest that a large fraction of male recombination in $D$. melanogaster occurs premeiotically (Hiraizumi et al., 1973; Kidwell and Kidwell, 1975b).

As previously mentioned, the data in table 2 suggest that in $O K 1 / d p b \mathrm{cn} b w$ males at least some recombination probably occurs in premeiotic germ cells. This is apparent from the recovery of presumptive clusters of double recombinants, which, if due to two single events, are expected to occur at a low frequency (about 0.002 per cent each). To determine whether other instances of recombination in $O K 1 / \mathrm{dp} b \mathrm{cn}$ bw males also occur in premeiotic cells, we performed an experiment in which single $O K 1 / d p b$ cn bw;OK1/ve males were mated with three $d p b c n$ bw;ve females in six 2-day broods or in four 4-day broods. Each male received fresh virgin females at each brood. This brood technique increases the number of progeny from each male, so that clusters of male recombinants, if produced, can readily be identified. 
The distribution of recombinant progeny from each male for each genetic interval $(d p-b, b-c n$, and $c n-b w)$ was tested for goodness-of-fit with the expected Poisson distribution by a determination of the index of dispersion $\chi^{2}$.

A total of 51 males were successfully carried through this brood programme and the results are summarised in table 4 . Forty-eight males gave recombinant progeny and the total recombination frequency $(406 / 22148=$ 1.83 per cent) is significantly higher $(P<0.001)$ than the control value $(1 / 53952=0.002$ per cent; table 2$)$.

Some clusters of male recombinants were recovered. For example, approximately one-third ( 27.9 per cent and 32.8 per cent) of the progeny

TABLE 4

Male recombination frequencies from individual $\mathrm{OK} 1$ males

\begin{tabular}{|c|c|c|c|}
\hline \multirow[b]{2}{*}{ Number of recombinations per male } & \multicolumn{3}{|c|}{$\begin{array}{c}\text { Number of males giving recombination } \\
\text { per region }\end{array}$} \\
\hline & $d p-b$ region & $b-c n$ region & $c n-b w$ region \\
\hline 0 & 38 & 19 & 9 \\
\hline I & 7 & 13 & 11 \\
\hline 2 & 3 & 6 & 10 \\
\hline 3 & 0 & 6 & 7 \\
\hline 4 & 1 & 4 & 4 \\
\hline 5 & i & 1 & 4 \\
\hline 6 & 0 & 0 & 4 \\
\hline 7 & 0 & 0 & 0 \\
\hline 8 & 1 & 1 & 0 \\
\hline 9 & 0 & 0 & 0 \\
\hline 10 & 0 & 0 & 0 \\
\hline 11 & 0 & 1 & 0 \\
\hline 69 & 0 & 0 & 1 \\
\hline 112 & 0 & 0 & 1 \\
\hline Total & 51 & 51 & 51 \\
\hline Total recombinations & 30 & 83 & 293 \\
\hline Total progeny & 22148 & 22148 & 22148 \\
\hline Recombination frequency $(\%)$ & $0 \cdot 14$ & 0.38 & 1.32 \\
\hline Mean & 0.588 & $1 \cdot 627$ & $5 \cdot 745$ \\
\hline Variance & $2 \cdot 127$ & 4.598 & $320 \cdot 874$ \\
\hline Index of dispersion $\chi_{50}^{2}$ & $180 \cdot 81$ & $141 \cdot 32$ & $2792 \cdot 59$ \\
\hline Probability & $P<0.001$ & $P<0.001$ & $P<0.001$ \\
\hline
\end{tabular}

of two different males were recombinants (in both cases $d p^{+} b^{+} c n^{+} b w$ ) suggesting early premeiotic events. It seems unusual to recover such a high frequency of recombination in males, but Hiraizumi et al. (1973) and Kidwell and Kidwell (1975b) have also observed comparatively large clusters of male recombinants. Moreover, as shown in table 4, the distributions of male recombinant events for each interval are significantly different from the expected Poisson distributions. This is true even if the two males which showed large clusters of recombinant progeny are excluded. These results suggest that at least some male recombination events in $O K 1 / d p b \mathrm{cn} b w$; $O K 1 / v e$ males are premeiotic in origin, although the possibility that male recombination also occurs in meiotic cells cannot be excluded. It also appears that recombination events in $O K 1 / d p b \mathrm{cn}$ bw males are restricted to germ cells. This is apparent from the recovery of only five flies in this entire 
study with mosaic spots in their eyes, an observation which is in contrast to that of Hiraizumi et al. (1973) for a south Texas line. In addition, no significant mitotic recombination has been observed in wings of $O K 1$ males (Thompson and Woodruff, in preparation).

The recovery of 48 males that showed recombination out of a total of $5 \mathrm{I}$ tested suggests that the male-recombination element in the $O K 1$ line is homozygous, and is, therefore, a dominant element that is homozygous viable. This was shown to be true when an isogenic $O K 1 / O K 1$ stock was synthesised by use of the balancer stock $\operatorname{In}(2 L R) C y / G l a$, and, as $O K 1 / d p b \mathrm{cn}$ bw males, was observed to show male recombination $(2 \mathrm{l} / 2472=0.85$ per cent $)$.

Finally, the results from the brood experiment suggest that there is no effect of age of the males on recombination in $O K 1 / d p b c n$ bw males. There is neither a significant increase nor decrease in the frequency of recombination with increasing male age. This is apparent from the similar male recombination frequencies observed among broods, with the assumption that large clusters are derived from single events.

(c) Chromosome interference. Because of the recovery of double recombination events in the $b$-bw region, it is possible to get a measure of chromosome interference for male recombination in this study. Yet, to get a realistic estimation of interference, any premeiotic recombination events that give rise to a cluster must be scored as an individual occurrence. Of course, it is impossible to identify with certainty all cluster events, even among the progeny of individual males. We have, therefore, determined a " conservative" estimation of interference for male recombination by the following procedure. From the progeny of any one male or from any one bottle (five males), we counted as a cluster any number of double recombination events above one, and we scored each cluster as an individual event. We also counted every single recombinant as an individual event. By this procedure, we may underestimate the true frequency of double male recombination events and overestimate the true frequencies of single events.

From the data in table 2, a total of 34 double recombinants were observed from a total of 13 bottles, and from the single-male brood experiment (table 4), two males gave one double recombinant each and one male gave three double recombinants. This gives, after considering possible clusters, a total of 16 double recombinants in the $b$-bw region out of 93,443 total flies $(0 \cdot 00017)$. The frequency of single recombinants between $b$ and $c n$ was $378 / 93443(0.00405)$, and the frequency between $c n$ and $b w$ was $623 / 93443$ $(0.00667)$. The observed frequency of double recombinants $(0.00017)$ was, therefore, six times higher than the expected frequency $(0.000027)$. Thus by our analysis, chromosome interference from recombination in $O K 1 / d p b \mathrm{cn}$ bw males is negative (coincidence of coefficient $=6 \cdot 3$ ). Negative interference has also been observed from recombination in males of Megaselia scalaris (Mainx and Doschek, 1967).

(d) Reciprocal-cross effect. Kidwell and Kidwell (1975a) and Sved (1976) have reported that a cytoplasmic-chromosomal interaction can influence the frequencies of male recombination and male or female sterility in $D$. melanogaster. This conclusion was based on significant differences in the frequencies of male recombination and male or female sterility for reciprocal crosses. Hinton (1974) reported a similar observation for male recombination in $D$. ananassae and proposed the presence of a cytoplasmic suppressor of male recombination. 
We have also observed a reciprocal-cross effect with the $O K 1$ line. Recombination was observed in the sons and sterility in the daughters of matings between marker females and $O K 1$ males, but not in the progeny of matings between $O K 1$ females and marker males. The mating scheme used in the reciprocal-cross experiment was as follows:

$$
\begin{aligned}
& \text { Cross } A \text { (male OK1 parent) } \\
& \text { G1 } d p b \text { cn bw;ve } \text { oᄋ } \times \text { - Single } O K 1 \quad \sigma^{\pi}
\end{aligned}
$$

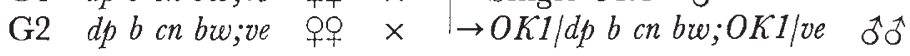

$$
\begin{aligned}
& \text { Cross } B \text { (female } O K 1 \text { parent) } \\
& \text { G1 Single } O K 1 \text { ㅇ } \times \text { - } d p b \text { cn bw;ve ỡ }
\end{aligned}
$$

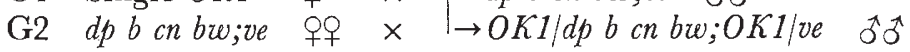

The G3 progeny of both crosses were scored for recombination. In addition, the fertility of G2 males and females from the reciprocal crosses was determined. Male fertility was scored by crossing single G2 males from each cross to three virgin $d p b \mathrm{cn} b w$; ve females. The fertility of females was tested by crossing single non-virgin G2 females with two sibs, and by crossing single virgin $\mathrm{G} 2$ females with two Canton- $S$ males. All vials were scored for the presence of larvae. Any female which gave one or more larvae was considered fertile.

Table 5 shows the male recombination results of these two crosses. There was a complete absence of male recombinants in G3 progeny of cross B, whereas male recombinants in G3 progeny of cross A occurred in a frequency that was not significantly different from other $O K 1$ crosses. Hence, only G2 progeny males from crosses of wild-type males with marker females showed recombination. These results are similar to those of Kidwell and Kidwell (1975a), Sved (1976), and Hinton (1974).

TABLE 5

Reciprocal-cross effect on male recombination (see text for details)

$\begin{array}{lcc} & \frac{\text { Recombinant progeny }}{\text { Total progeny }} & \begin{array}{c}\% \text { Male } \\ \text { recombination }\end{array} \\ \text { Cross A (male } O K 1 \text { parent) } & 63 / 8541 & 0 \cdot 74 \\ \text { G2 males } & 67 / 6033 & 1 \cdot 11 \\ \text { G3 males } & 66 / 5262 & 1 \cdot 25 \\ \text { G4 males } & & \\ \text { Cross B (female } O K 1 \text { parent) } & 0 / 9481 & 0 \cdot 0 \\ \text { G2 males } & 54 / 7093 & 0 \cdot 76 \\ \text { G3 males } & 28 / 3437 & 0 \cdot 81 \\ \text { G4 males } & & \end{array}$

To determine whether this reciprocal cross pattern of male recombination was present in subsequent generations, OK1/dp $b$ cn bw G3 and G4 male progeny of both crosses were mated to $d p b$ cn bw; ve females and progeny were scored for male recombination. Male recombination was then observed at similar frequencies in the G3 and G4 progeny of both cross A and cross B (table 5); a result that is similar to that of Kidwell and Kidwell $(1975 a)$. 
The observation that the cross-B G3 and G4 males showed recombination rules out the possibility that the $O K 1 \mathrm{Y}$ chromosome is responsible for the reciprocal-cross effect. We tested the possibility that the reciprocal-cross effect was caused by a cytoplasmic suppressor of male recombination in the $O K 1$ line by the mating scheme in fig. 2. In this scheme, the cytoplasm of the Gl OK 1 female is transferred to the G5 $d p b$ cn bw; ve females. If there is a cytoplasmic suppressor of male recombination in the $O K 1$ stock, and if the suppressor is not diluted out in five generations, this suppressor should be present in the G6-A males, where it would suppress male recombination and cause a reciprocal-cross effect. The cytoplasm in the G6-B males is from

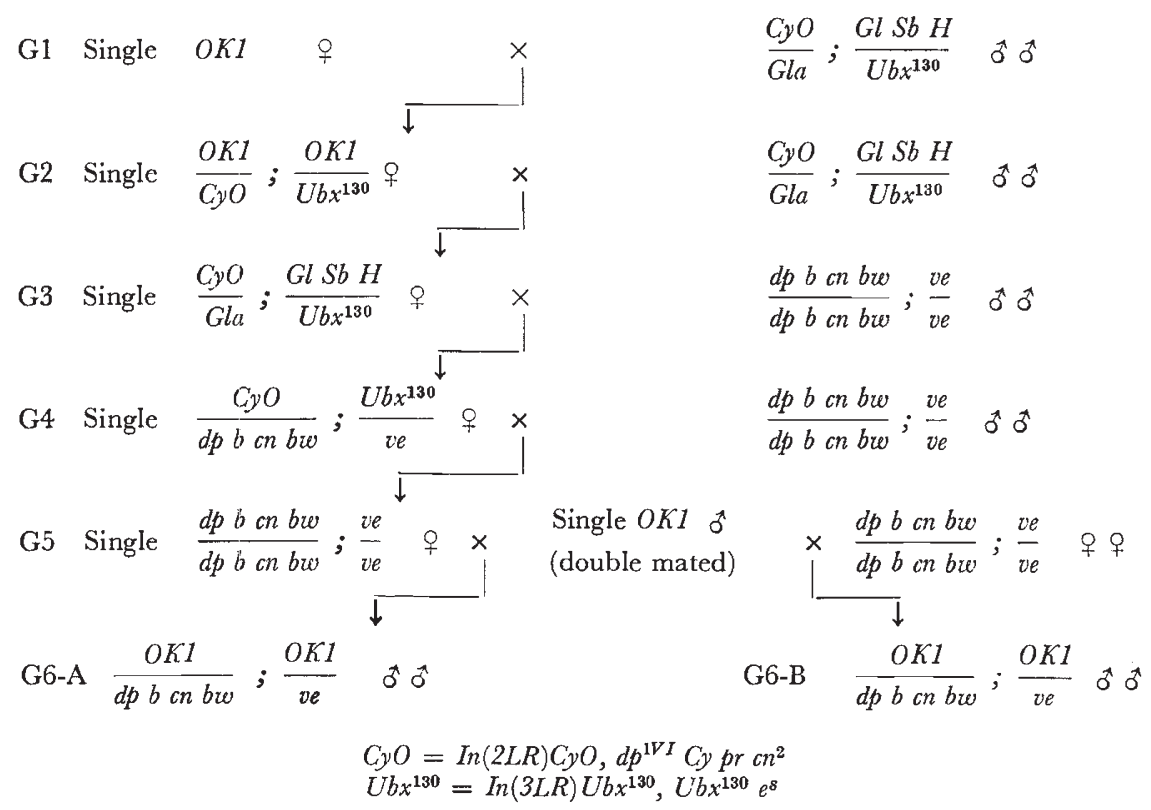

FIG. 2.-Mating scheme to determine if there is a cytoplasmic suppressor of male recombination in $O K 1$

the $d p b c n b w ; v e$ stock, and it would not be expected to induce a reciprocalcross effect. The G6-B cross, therefore, serves as a control for male recombination.

Two different Gl females (Gl $\&$ No. 1 and Gl $q$ No. 2) were placed into these crosses. Both G6-A and G6-B males were crossed to $d p b$ cn bw; ve females, and G7 progeny were scored for recombination. The results of these crosses are shown in table 6 . There is a lower frequency of male recombination in cross $6-\mathrm{B}$, but this difference is not significant at the 5 per cent level $\left(\chi^{2}=3 \cdot 2\right)$. There does not seem, therefore, to be a cytoplasmic suppressor of male recombination in the $O K 1$ line. However, these data do not rule out the possibility that $O K 1$ chromosomes produce a suppressor of male recombination.

As shown in table 7, unlike the results of Kidwell and Kidwell (1975a), there was no significant difference in the frequencies of Fl male sterility in these reciprocal crosses. On the other hand, in agreement with Kidwell 
and Kidwell (1976) and Sved (1975a), there was a significant difference in the frequencies of $\mathrm{F} 1$ female sterility in these reciprocal crosses. F1 females from cross $\mathrm{A}$, whether crossed to sibs or to Canton-S males, showed a significantly higher frequency of sterility than did females of cross B. Picard (1976) has observed a similar pattern of female sterility in D. melanogaster.

TABLE 6

Test for the presence of a cytoplasmic suppressor of male recombination in OK1 (see fig. 2 and text for details)

\begin{tabular}{|c|c|c|}
\hline & Recombinant progeny & $\%$ \\
\hline & Total progeny & recoml \\
\hline Cross 6-A $(O K)$ & & \\
\hline G1 우 No. 1 & $19 / 1590$ & \\
\hline G1 오 No. 2 & $14 / 1297$ & \\
\hline Total & $33 / 2887$ & \\
\hline Cross 6-B $(d p b$ & & \\
\hline G1 우 No. 1 & $4 / 1253$ & \\
\hline G1 $\&$ No. 2 & $10 / 993$ & \\
\hline Total & $14 / 2246$ & \\
\hline
\end{tabular}

TABLE 7

Reciprocal-cross effect on male and female fertility (see text for details)

$\begin{array}{lc} & \begin{array}{c}\text { Sterile matings/total matings } \\ (\% \text { sterility) }\end{array} \\ \text { Cross A (male } O K 1 \text { parent) } & 13 / 100(13.0 \%) \\ \text { G2 males } & 24 / 116(20 \cdot 7 \%)^{\mathrm{a}} \\ \text { G2 females (mated to sibs) } & 46 / 123(37 \cdot 4 \%)^{\mathrm{b}} \\ \text { G2 females (mated to Canton-S) } & \\ \text { Cross B (female } O K 1 \text { parent) } & 14 / 99(14 \cdot 1 \%) \\ \text { G2 males } & 4 / 107(3 \cdot 7 \%)^{\mathrm{a}} \\ \text { G2 females (mated to sibs) } & 7 / 119(5 \cdot 9 \%)^{\mathrm{b}} \\ \text { G2 females (mated to Canton-S) } & \\ \text { a } \chi_{1}^{2}=13 \cdot 1, P<0 \cdot 01 & \\ \text { b } \chi_{1}^{2}=33 \cdot 3, P<0 \cdot 001 & \end{array}$

\section{Discussion}

The above data show that male recombination is present in stocks recently derived from natural populations of D. melanogaster from Oklahoma, U.S.A., and from Cambridge, England. These populations add to the growing world-wide list of populations that exhibit male recombination.

Why, then, was male recombination not reported before 1971? This was probably due to several reasons. Male recombination would not be observed in most experiments because of its low frequency, and because of inappropriate mating schemes. Those rare male recombinations events that did occur were probably classified as the products of mutation. In addition, it has been reported that male recombination does not seem to occur in stocks that have been in the laboratory for a number of years, and some populations do not show male recombination. 
Even with these reasons taken into consideration, the early Drosophila workers should have observed male recombination, and we believe that in some cases they did. Muller (1916), and Bridges and Morgan (1919) reported the recovery of progeny that were probably the result of spontaneous male recombination. For example, from the progeny of one $++1 \mathrm{pr} v g$ male mated to $p r v g / p r v g$ females, Bridges and Morgan (1919) observed in June 1912, in one of the first backcrosses performed with linked autosomal mutations: 192 pr vg/pr vg, $338++/ p r v g, 31$ pr $+/ p r v g$, and $5+v g / p r v g$ progeny. This gives a frequency of male recombination of 6.4 per cent between $p r$ and $v g$, two markers that are 12.5 female-derived map units apart (Lindsley and Grell, 1968).

Now that it is known that male recombination occurs in $D$. melanogaster, we need to answer three important questions: (1) What is the genetic basis of male recombination? (2) What is its mode of action? (3) Does male recombination affect the adaptability of natural populations?

An answer to the last question awaits a more detailed determination of the frequencies of male recombination within natural populations. Indeed, we do not even know whether male recombination occurs in natural populations, we only know that it occurs in the laboratory among outcrossed males. It has been postulated that in some populations there is a close correlation among male recombination and a distortion segregation frequency (Hiraizumi, 1971), chromosome aberrations (Voelker, 1974; Yamaguchi and Mukai, 1974; Yamaguchi, 1976), mutator activity (Slatko and Hiraizumi, 1973; Kidwell and Kidwell, 1975b; Woodruff and Thompson, 1975), and sterility (Kidwell and Kidwell, 1975a; Sved, 1976). All of these events could increase genetic variability.

The results of this and other studies (Hiraizumi, 1971; Slatko and Hiraizumi, 1973; Waddle and Oster, 1974; Yamaguchi, 1976) suggest that in some natural populations the frequencies of chromosomes with male recombination potential are high-20-50 per cent or more. These populations might have high enough frequencies of male recombination, and associated events, to influence their adaptability. On the other hand, it is known that a few natural populations do not show male recombination (Slatko and Hiraizumi, personal communication).

The phenomenon of male recombination in the $O K 1$ line is similar to that reported for other natural population lines. There are dominant elements on the second and on the third chromosomes that induce both single and multiple male recombination events. One element alone can induce male recombination, but the highest frequencies of recombination are induced when both elements are present. These recombination events occur mainly in the centromeric region and in the right arm, and there is a reciprocal-cross effect associated with male recombination and female sterility.

The results of this study also show that some male recombinants occur in large clusters. This suggests that at least some male recombination events occur in spermatogonia. Hiraizumi et al. (1973), and Kidwell and Kidwell (1975b) have also recovered clusters of male recombinants, and we believe that Muller (1916), and Bridges and Morgan (1919) did, too. Furthermore, spontaneous $\mathrm{X}-\mathrm{Y}$ recombination in males has been reported to occur as clusters (Lindsley, 1955), and recombination in males of Megaselia scalaris occurs in spermatogonia (Mainx and Doschek, 1967). In contrast, there 
is no firm evidence for the premeiotic origin of male recombination in $D$. ananassae (Kale, 1969).

It is not known if male recombination events in $D$. melanogaster can also occur in meiotic cells. Hinton and Downs (1975) have tested for male recombination in spermatocytes of $D$. ananassae by scoring for the presence of anaphase bridges and fragments produced by male recombination within heterozygous inversions. No true bridges and fragments were observed. We are currently performing a similar test in $D$. melanogaster.

We stated that we would use "male recombination" to mean exchange of markers without specifying a mechanism of exchange. Male recombination events could occur by classical crossing over, as in females, or by chromosome breakage and reunion events. There is some evidence which suggests that male recombination does not occur by the same mechanism as female recombination, implying that male recombination is not occurring by classical crossing over. (I) A large proportion of male recombination events occur within centromeric regions, whereas this is not the case in females. (2) Chromosome interference appears to be negative for male recombination. (3) There is no interchromosomal effect on male recombination (Woodruff, Slatko and Thompson, in preparation). (4) There is a failure to recover reciprocal recombination events in some regions. We believe, therefore, that, although the evidence is inconclusive, male recombination probably occurs by breakage and reunion of chromosomes. Slatko and Hiraizumi (1973), Voelker (1974) and Yamaguchi (1976) have previously postulated that chromosome breakage is associated with male recombination.

Chromosome breakage is certainly associated with male recombination in some strains (Voelker, 1974; Yamaguchi and Mukai, 1974; Yamaguchi, 1976), and we have evidence that this is occurring in outcrossed $O K 1$ males (Woodruff, Henderson and Thompson, in prep.). If male recombination does occur by chromosome breakage and reunion the breaks could occur at different positions within the two exchange chromosomes resulting in one recombinant chromosome with a deficiency and one with a duplication. Some of these chromosomes would be homozygous lethal, depending on the site and location of the aberrations. On the other hand, if male recombination occurs by classical crossing over, whether mitotic or meiotic, an exact exchange of genetic material would occur and the exchange products would be homozygous viable. Thus an analysis of the viability of homozygous male recombinant chromosomes should help to determine which of these two possible mechanisms of male recombination is correct. These studies are now in progress.

It has been shown in this and and in other studies (Kidwell and Kidwell, 1975a, Bucheton et al., 1976; Picard, 1976; Sved, 1976) that male or female sterility occurs in the progeny of some outcross natural population lines of $D$. melanogaster, and that these lines often also show male recombination; this phenomenon has been called hybrid dysgenesis by Kidwell and Kidwell (1976), and by Sved (1976). A similar observation of hybrid male sterility has been made for strains of $D$. paulistorum (Williamson et al., 1971). In this latter case, it has been proposed that an intracellular mycoplasma-like organism may cause this sterility; it would be of interest to determine if this organism also affects recombination. The element in $D$. melanogaster that causes sterility is unknown, but it may also be a micro-organism. 
With this in mind, it has been postulated that episomes (Voelker, 1974; Waddle and Oster, 1974), or viruses (Roberts, 1976) may be responsible for male recombination induction. We believe that there are observations that support these hypotheses: (1) the reciprocal-cross effect on male recombination; (2) Sochacka and Woodruff (1976) have induced recombination in Canton-S/dp $b c n$ bw males by injections with whole-fly or ovarian extracts from a male recombination line $(O K 1)$. It is possible that microorganisms in these lines induce chromosome breaks which lead to male recombination and sterility.

Finally, many laboratory stocks do not show male recombination. This may be because these stocks never did show male recombination, or because they have lost the ability to undergo male recombination. It is of interest, therefore, to determine if the frequency of male recombination in the $O K 1$ line has been affected by keeping it in a laboratory environment for a number of years. The $O K 1$ line, which was originally derived from a single female, has been kept by mass transfer every 2 weeks since its isolation in October 1970 (this stock was first tested for male recombination in October 1974). In May 1976, after approximately 140 generations, the $O K I$ line was again tested for male recombination. Ten individual $O K 1 / O K 1$ males were mated to $d p b$ cn $b w ; v e$ females and $O K 1 / d p b c n b w ; O K 1 / v e$ progeny males from each cross were mated to $d p b c n$ bw; ve females. Male recombination was observed among nine of the ten matings for a total frequency of 3.54 per cent (238/ 6726). Thus, after almost $5 \frac{1}{2}$ years in the laboratory, the $O K 1$ line still shows very high levels of male recombination.

Acknowledgment.-We are grateful to Professor J. M. Thoday, Drs M. Ashburner, Y. Hiraizumi, J. Holden, G. Richards, and B. Slatko for their advice and critical review of the manuscript. We also thank Mrs A. Tomsett for her technical assistance.

Part of this work was carried out during the tenure of grants from the S.R.C. of Great Britain to Professor Thoday (J. N. T.) and the M.R.C. of Great Britain to Dr M. Ashburner and Professor B. Clark (R. C. W.). We also gratefully acknowledge support from a Faculty Research Grant from the University of Oklahoma to J. N. T. and N.I.H. grant 1-R01ES01439-01.

\section{ReFERENCES}

BRIDGes, C. B., AND MORGAN, T. H. 1919. Contributions to the genetics of Drosophila melanogaster. II. The second chromosome group of mutant characters. Carnegie Inst. Wash. Publ., 278, 125-304.

Bucheton, A., LAVige, J. M., Picard, G., AND heritier, Ph. 1976. Non-Mendelian female sterility in Drosophila melanogaster: Quantitative variations in the efficiency of inducer and reactive strains. Heredity, 36, 305-314.

Franca, z. M., DA CUNHA, A. B., AND GARRIDO, M. c. 1968. Recombination in Drosophila willistoni. Heredity, 23, 199-204.

HiNTON, c. w. 1974. An extrachromosomal suppressor of male crossing over in Drosophila ananassae. In Mechanisms in Recombination, ed. R. F. Grell, pp. 391-397. Plenum, New York.

hinton, c. w., AND Downs, J. E. 1975. The mitotic, polytene, and meiotic chromosomes of Drosophila ananassae. 7 . Heredity, 66, 353-361.

HIRAIZUmi, y. 1971. Spontaneous recombination in Drosophila melanogaster males. Proc. Nat. Acad. Sci., U.S.A., 68, 268-270.

hiRAizumi, Y., SlAtKo, B., LANGley, G., AND NILL, A. 1973. Recombination in Drosophila melanogaster male. Genetics, 73, 439-444.

KALE, P. G. 1969. The meiotic origin of spontaneous crossovers in Drosophila ananassae males. Genetics, 62, 123-133.

KIDWELL, M. G., AND Kidwell, J. F. 1975a. Cytoplasm-chromosome interactions in Drosophila melanogaster. Nature, 253, 755-756. 
KIDWELL, M. G., AND KIDWELl, J. F. 1975b. Spontaneous male recombination and mutation in isogenic-derived chromosomes of Drosophila melanogaster. 7. Heredity, 66, 367-375.

kidwell, M. G., AND KidWell, J. F. 1976. Selection for male recombination in Drosophila melanogaster. Genetics, 84, 333-351.

KIKkAWA, H. 1935. Crossing-over in the male of Drosophila virilis. Cytologia, 6, 190-194.

LINDSLEY, D. L. 1955. Spermatogonial exchange between the $\mathrm{X}$ and $\mathrm{Y}$ chromosomes of Drosophila melanogaster. Genetics, 40, 24-44.

LINDSLEY, D. L., AND GRELL, E. H. 1968. Genetic variations of Drosophila melanogaster. Carnegie Inst. Wash. Publ. 627.

maInX, R. 1964. The genetics of Megaselia scalaris Loew (Phoridae): A new type of sex determination in Diptera. Amer. Nat., 98, 415-430.

MAINX, R., AÑ DOSGHEK, E. 1967. Der genetische austausch bei den beiden geschlechtern von Megaselia scalaris. Molec. Gen. Genetics, 99, 203-218.

MORGAN, T. H. 1912. Complete linkage in the second chromosome of the male of Drosophila. Science, 36, 718-720.

MORGAN, T. H. 1914. No crossing over in the male of Drosophila of genes in the second and third pairs of chromosomes. Biological Bulletin, 26, 195-204.

MORIWAKI, D., AND tobari, Y. N. 1975. Drosophila ananassae. In Handbook of Genetics, vol. 3, ed. R. C. King. Plenum Press, New York.

MULLER, H. J. 1916. The mechanism of crossing over. IV. The manner of occurrence of crossing over. Amer. Nat., 50, 284-305.

PHILlips, U. 1944. Crossing over in the males of Drosophila subobscura. Nature, 153, 223.

PICARD, G. 1976. Non-mendelian female sterility in Drosophila melanogaster: Hereditary transmission of I factor. Genetics, 83, 107-123.

ROBERTS, P. A. 1976. The genetics of chromosome aberration. In The Genetics and Biology of Drosophila, vol. la, eds. M. Ashburner and E. Novitski. pp. 67-184.

SLATKO, B. E., AND HIRAIZUMI, Y. 1973. Mutation induction in the male recombination strains of Drosphila melanogaster. Genetics, 75, 643-649.

SlAtKo, B. E., AND HiRAizUmi, Y. 1975. Elements causing male crossing over in Drosophila melanogaster. Genetics, 81, 313-324.

SOCHACKA, J. H. N., AND WOODRUFF, R. C. 1976. Induction of male recombination in Drosophila melanogaster by injection of extracts of flies showing male recombination. Nature, 262, 287-289.

SVED, J. A. 1974. Association between male recombination and rapid mutational changes in Drosophila melanogaster. Genetics, 77, s64.

sved, J. A. 1976. Hybrid dygenesis in Drosophila melanogaster: A possible explanation in terms of spatial organisation of chromosomes. Australian Journal of Biological Science, 29, 375-388.

VOELKER, R. A. 1974. The genetics and cytology of a mutator factor in Drosophila melanogaster. Mutation Research, 22, 265-276.

WADDLE, F. R., AND OSTER, I. I. 1974. Autosomal recombination in males of Drosophila melanogaster caused by a transmissible factor. F. Genetics, 61, 177-183.

WILliamson, D. L., EHRMAN, I.., AND KERNAGHAN, R. P. 1971. Induction of sterility in Drosophila paulistorum: Effect of cytoplasmic factor. Proc. Nat. Acad. Sci., U.S.A., 68, 2158-2160.

WOODRUFF, R. C., AND BORTOLOzz1, J. 1976. Spontaneous recombination in males of Drosophila simulans. Heredity, 37, 295-298.

WOODRUFF, R. C., AND THOMPSON, J. N. JR. 1975. Genetic analysis of male recombination in Drosophila melanogaster. Genetics, 80, s86.

YAMAGUCHI, O. 1976. Spontaneous chromosome mutation and screening of mutator factors in Drosophila melanogaster. Mutation Research, 34, 389-406.

YAMAGUCHI, O., AND MUKAI, T. 1974. Variation of spontaneous occurrence rates of chromosomal aberrations in the second chromosomes of Drosophila melanogaster. Genetics, 78, 1209-1221. 